\title{
Monitoramento Analítico do Plano Brasil Sem Miséria e de Programas do Ministério do Desenvolvimento Social e Combate à Fome
}

Marconi Fernandes de Sousa ${ }^{1}$
O presente relato tem por objetivo apresentar a sistemática de produção e disseminação dos principais indicadores de monitoramento relativos aos programas e ações do Plano Brasil Sem Miséria (BSM) e do Ministério de Desenvolvimento Social e Combate à Fome (MDS) organizados pelo Departamento de Monitoramento (DM). Para tanto, o relato apresenta breves descrições das atividades operacionais e diárias de extração de dados, construção de indicadores, assim como de concepção e desenvolvimento de plataformas de disseminação desses indicadores.

\section{Monitoramento de Programas e os Indicadores}

A institucionalização das atividades de monitoramento e avaliação (M\&A) da ação governamental vem ganhando força no Brasil, acompanhando o esforço de ampliação do escopo e escala dos programas sociais. Recursos crescentes são aportados no levantamento de informações para gestão e aprimoramento de programas no País, assim como no aumento da qualificação média dos técnicos e gestores nas três esferas do setor público.
1 Diretor de Monitoramento da Secretaria de Avaliação e Gestão da Informação do Ministério de Desenvolvimento Social e Combate à Fome. 
No MDS há um efetivo esforço nesse sentido, que já data de sua criação em 2004, quando do estabelecimento em seu organograma da Secretaria de Avaliação e Gestão da Informação (SAGI). As atividades de monitoramento foram potencializadas com a criação do Departamento de Monitoramento (DM), em 2010, no reconhecimento da necessidade de se trabalhar analiticamente com as informações geradas tanto pelos sistemas de gestão do Ministério como com as demais informações produzidas pelo Sistema Estatístico Nacional. E, neste sentido, assinala-se a importância de gerar indicadores de monitoramento mais específicos e periódicos para acompanhamento das atividades envolvidas.

Com a criação do Plano Brasil Sem Miséria em 2011, em virtude de sua abrangência e da criticidade de muitas de suas ações, tem-se exigido das atividades do Departamento de Monitoramento a estruturação de procedimentos de cômputo periódico de indicadores sobre diferentes temáticas e domínios territoriais para acompanhamento de metas e para análise dos desembolsos financeiros, de realização de atividades-meio, de entrega de produtos e de inferência de re- sultados dos programas junto aos seus públicos-alvo.

Partindo da lógica de intervenção dos programas e ações, é necessário dispor-se de medidas que permitam acompanhar o esforço governamental da alocação de recursos aos possíveis efeitos na sociedade. Tal como termômetros ou sismógrafos, os indicadores de monitoramento se prestam a medir a "saúde do paciente" ou "estabilidade do território" e antecipar com alguma presteza as informações cruciais para correção de rumos ou ações contingenciais.

Um bom conjunto de indicadores de monitoramento - organizado em um sistema ou em uma proposta mais pragmática em painel - não é necessariamente composto de grande quantidade de informação, mas sim de um sistema em que a informação foi selecionada de diferentes fontes e está organizada de forma sintetizada e mais adequada ao uso analítico pelos diferentes gestores. Busca-se encontrar um ponto de equilíbrio entre o "caos informacional", potencialmente gerado pela estruturação de sistemas de monitoramento construídos de baixo para cima (em que participam inicialmente técnicos e gestores da 
base e depois de níveis táticos e mais estratégicos), e a pobreza analítica das propostas desenvolvidas de cima para baixo.

O relato descreve um conjunto de esforços para consolidação de um sistema de monitoramento que reúne informações sintéticas - para análise de tendências gerais das atividades estratégicas - e informações analíticas - para entendimento mais aprofundado das tendências observadas. O esforço veio acompanhado de algumas premissas básicas para escolha de indicadores, prezando-se pela análise de sua aderência às propriedades de relevância social, validade de constructo, confiabilidade, periodicidade, sensibilidade às mudanças e especificidades das ações programadas.

\section{Os Indicadores de Monitoramento na SAGI}

O Departamento de Monitoramento da SAGl é a unidade responsável pela construção de diversos indicadores para o Plano Brasil Sem Miséria. Valendo-se de diferentes fontes de dados - Censos Demográficos, edições da Pesquisa Nacional por Amostra de Domicílios (PNAD), Cadastro Único para Programas Sociais (Cadúni-
Co), Relação Anual de Informações Sociais (RAIS), registros de programas do MDS e outros ministérios e outras pesquisas do Sistema Estatístico Nacional - e variados procedimentos estatísticos e integração de dados, o Departamento tem criado um sistema com conjunto amplo de indicadores que permitem aos gestores dos três níveis do governo acompanhar as ações do Plano com grande detalhe temático e geográfico.

Dessa forma, a concepção e desenvolvimento das ferramentas de monitoramento do DM foram ancorados primeiramente na definição da estrutura/arquitetura do banco de dados no que concerne às granularidades temporais e territoriais dos indicadores que iriam compô-lo; segundo, na definição de uma metodologia de documentação sintética e acessível dos metadados e, por fim, no estabelecimento da manutenção da memória das rotinas de extração, construção e carga de indicadores para fins de angariar eficiência na sua atualização. Afinal, um sistema de indicadores de monitoramento deve subsidiar tempestivamente os gestores nos processos de acompanhamento e análise dos indicadores afetos à performance das ações e programas. 


\section{QUUADRO 1: RELAÇÃO DAS PRINCIPAIS BASES DE DADOS TRABALHADAS NO DEPARTAMENTO DE MONITORAMENTO SEGUNDO FONTE}

\section{FONTES DE DADOS}

ANEEL, Dados agregados de beneficiários da tarifa social de energia elétrica

ANVISA, Programa de Análise de Resíduos de Agrotóxicos em Alimentos (PARA)

ANVISA, Programa de Análise de Resíduos de Medicamentos Veterinários em Alimentos de Origem Animal (PAMVET) CAIXA, Cadastro Único para Programas Sociais (CadÚnico)

CAIXA, Folha de Pagamentos do Programa Bolsa Família (PBF)

CONAB, Programa Brasileiro de Modernização do Mercado Hortigranjeiro (PROHORT)

DIEESE, Pesquisa Nacional da Cesta Básica de Alimentos

IBGE, Censo Agropecuário

IBGE, Censo Demográfico

IBGE, Contas Nacionais / Produto Interno Bruto dos Municípios

IBGE, Pesquisa da Pecuária Municipal

IBGE, Pesquisa de Informações Básicas Municipais (MUNIC)

IBGE, Pesquisa de Orçamentos Familiares (POF)

IBGE, Pesquisa Nacional por Amostra de Domicílios (PNAD)

IBGE, Produção Agrícola Municipal (PAM)

IBGE, Produção da Extração Vegetal e da Silvicultura

IBGE, Sistema Nacional de Índices de Preços ao Consumidor (INPC e IPCA) e Índices de Preços ao Produtor (IPP)

INEP, Censo da Educação Superior

INEP, Censo Escolar

MDA, Folha de Pagamento do Programa de Fomento às Atividades Produtivas Rurais

MTE, Relação Anual de Informações Sociais (RAIS)

- figura 1: Gestor de metadados e carGa de dAdos

\begin{tabular}{|c|c|c|c|c|c|c|c|c|}
\hline \multicolumn{4}{|c|}{ Sistema de Consultas } & \multicolumn{2}{|c|}{ UPLOAD DE DADOS } & 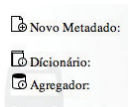 & \multicolumn{2}{|c|}{$\begin{array}{l}\text { (BSM) (BSM2) (PEPE } \\
\text { (BSM) (BSM2) (PEPE } \\
\text { (BSM) (BSM2) (PEPE }\end{array}$} \\
\hline Nome da $T$ & bela: & & & ID Tabela: & Tema & las: TODOS & $\because$ & \\
\hline Nome Vari & & & & ID Variáveis: & & & & \\
\hline Metadado & $\begin{array}{l}\text { Atualizar } \\
\text { esso ao }\end{array}$ & $\begin{array}{r}\text { Banco } \\
\text { form }\end{array}$ & $\begin{array}{l}\text { ID } \\
\text { lário }\end{array}$ & Tabela & Variáveis & ID Vars & Ultim? & $\begin{array}{l}\text { Visualizar } \\
\text { Dados }\end{array}$ \\
\hline 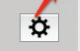 & \pm & BSM & 1 & $\begin{array}{l}\text { Quantidade de vínculos empregatícios em } \\
\text { ocupaçôes formais }\end{array}$ & 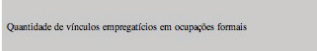 & v1 & 31 meses & $\odot$ \\
\hline 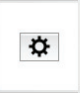 & \pm & BSM & 2 & $\begin{array}{l}\text { Quantidade de vínculos empregatícios em } \\
\text { ocupaçōes formais por setor econômico }\end{array}$ & 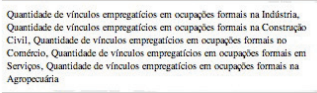 & $\begin{array}{l}\text { v2. v3, v4, v5. } \\
\text { v6 }\end{array}$ & 31 meses & $\odot$ \\
\hline 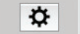 & \pm & BSM & 3 & Admissões, desligamentos e saldo & Admissóses, Desiligancentss, Saldo & v7, $88, v 9$ & 2 meses & $\odot$ \\
\hline$\$$ & \pm & BSM & 4 & $\begin{array}{l}\text { Total, entradas, desligamentos e saldo de famflias } \\
\text { beneficiárias do PBF }\end{array}$ & 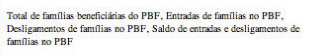 & $\begin{array}{l}\text { v10, v11, v12, } \\
\text { v13 }\end{array}$ & 10 meses & $\odot$ \\
\hline$\$$ & \pm & BSM & 5 & População total (Censo) & Populasán total & v14 & 43 meses & $\odot$ \\
\hline
\end{tabular}

Revista Brasileira de Monitoramento e Avaliação | Número 5 | Janeiro-Junho de 2013

Monitoramento Analítico do Plano Brasil Sem Miséria e de Programas do Ministério do Desenvolvimento Social e Combate à Fome 
Partindo da arquitetura do banco de dados da Matriz de Informações Sociais, o DM estruturou um banco de dados ampliado em sua estrutura de tabelas com a inclusão da granularidade nacional e regional, e desenvolveu uma metodologia de extração, geração e carga de indicadores que permitisse uma interação acessível e inteligível aos seus técnicos nas rotinas de alimentação do banco o $\checkmark$ que, consequentemente, tornou eficien- te a análise de conteúdo e pertinência dos indicadores nas aplicações de monitoramento. Com uma equipe majoritariamente composta de técnicos e consultores especialistas em indicadores, era necessária a consolidação das rotinas de trabalho voltadas à análise de indicadores e menos nas rotinas de carga. 0 Quadro 1 apresenta a relação das principais fontes de dados trabalhadas no Departamento.

- FIGURA 2: FORMULÁRIO DE DOCUMENTAÇÃO DOS METADADOS DA TABELA

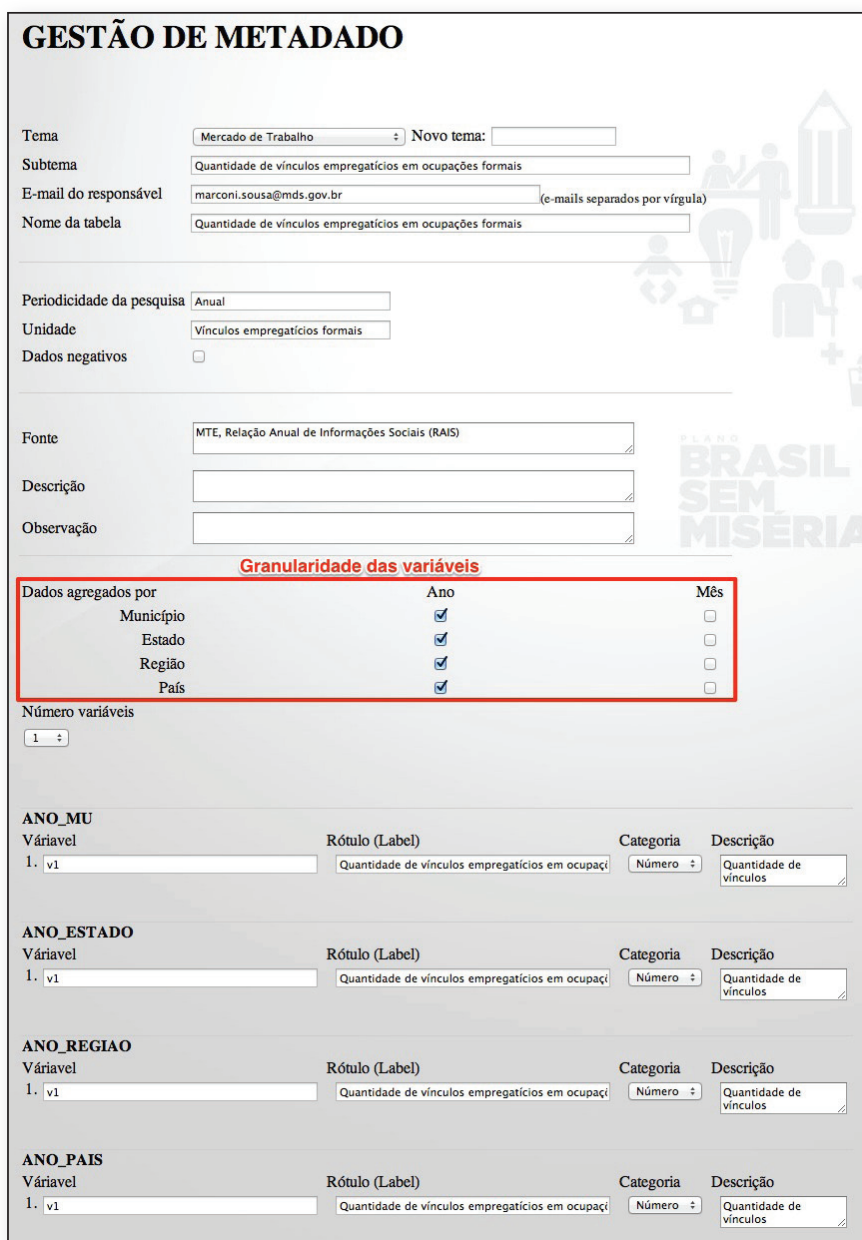


O gerenciamento do banco de dados é feito por meio de uma aplicação de gestão/documentação de metadados e carga de dados. Na aplicação, as variáveis estão distribuídas em tabelas e estas estão documentadas em formulários compostos pelas informações relativas ao tema, periodicidade, unidade de análise, fonte, descrição, observações adicionais, agregações temporais e territoriais e categoria das variáveis que a compõem (Ver Figuras 1 e 2).

É importante ressaltar que o sistema de sinaleiras apresenta a distância temporal para a data da última atualização da tabela, mas não leva em consideração os cronogramas de disponibilização dos registros/pesquisas de suas respectivas instituições produtoras. Dessa forma, funciona como informação adicional para alguns dados (exemplo: Censo Demográfico do IBGE, pesquisa decenal) e para dados mensais funciona como sistema de alerta para atualização dos dados (exemplo: Cadastro Geral de Empregados e Desempregados - CAGED do MTE).

O processo de carga é feito por meio de arquivos texto (comma separated values - extensão (sv), que são compostos por colunas com o código das unidades territoriais utilizadas pelo IBGE, mês, ano e as variáveis da tabela. Cada tabela tem seu respectivo arquivo texto e a aplicação espelha estas informações no banco de dados que está em formato postgres. Dessa forma, toda interação dos técnicos do DM com as atividades de carga de dados pode ser feita por meio de planilhas eletrônicas (Exemplo: Excel, Open Office, Numbers, etc.).

\section{- FIGURA 3: VISUALIZADOR DE DADOS}

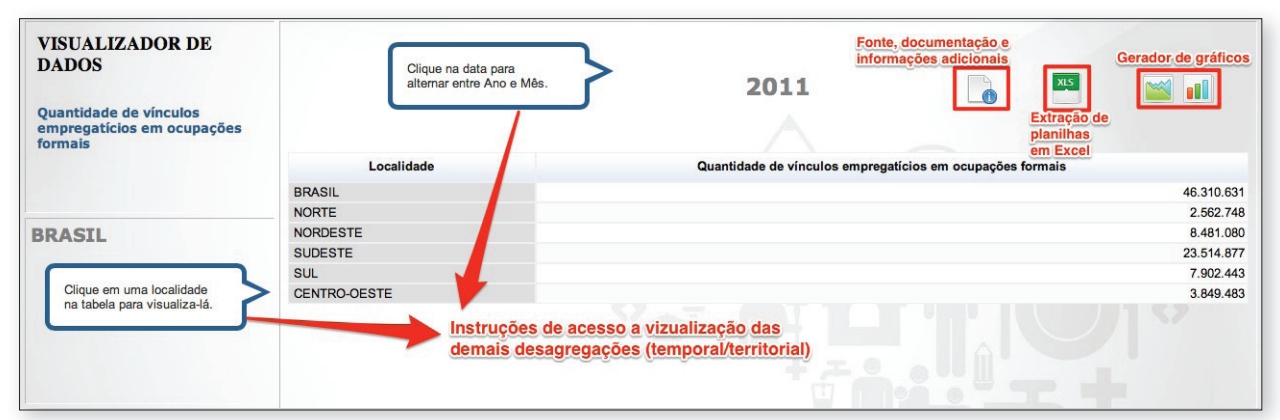

Revista Brasileira de Monitoramento e Avaliação | Número 5 | Janeiro-Junho de 2013 


\section{TABELA 1: DISTRIBUIÇÃO DAS VARIÁVEIS/INDICADORES DO BANCO DE DADOS DO DEPARTAMENTO DE MONITORAMENTO SEGUNDO ÁREA TEMÁTICA}

\section{ÁREA TEMÁTICA}

u

(1)

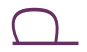

(1)

$\circlearrowright$

u

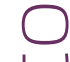

$+$

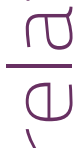

\begin{tabular}{lc|}
\hline Assistência Social & 195 \\
\hline Busca Ativa & 2 \\
\hline Condicionalidades & 61 \\
\hline Demografia & 90 \\
\hline Desigualdade & 55 \\
\hline Economia & 63 \\
\hline Educação & 204 \\
\hline Habitação & 108 \\
\hline Inclusão Produtiva & 18 \\
\hline Mercado de Trabalho & 225 \\
\hline Saúde & 76 \\
\hline Segurança Alimentar e Nutricional & 351 \\
\hline Transferência de Renda & 208 \\
\hline Total & 1656 \\
\hline
\end{tabular}

Por fim, a aplicação tem o módulo de visualização da tabela com funcionalidades de geração de gráficos e extração de planilhas.

Definida a arquitetura de banco de dados e os procedimentos para sua alimentação, cada tabela do banco tem sua respectiva documentação relativa aos procedimentos da rotina de extração e cálculo de seus indicadores/variáveis. Estas rotinas são desenvolvidas ou a partir dos microdados
QUANTIDADE DE VARIÁVEIS/INDICADORES

195

dos registros/pesquisas ou a partir de extrações de indicadores já calculados pelas instituições produtoras em seus canais oficiais de disseminação. As rotinas realizadas com os microdados são documentadas em textos instrucionais definindo etapas do processo e localização física dos arquivos nos computadores do Departamento, assim como em scripts/sintaxes de programação de aplicações de softwares de estatística. 
FIGURA 4: FLUXOGRAMA DA SISTEMÁTICA DE MONITORAMENTO DO DEPARTAMENTO DE MONITORAMENTO

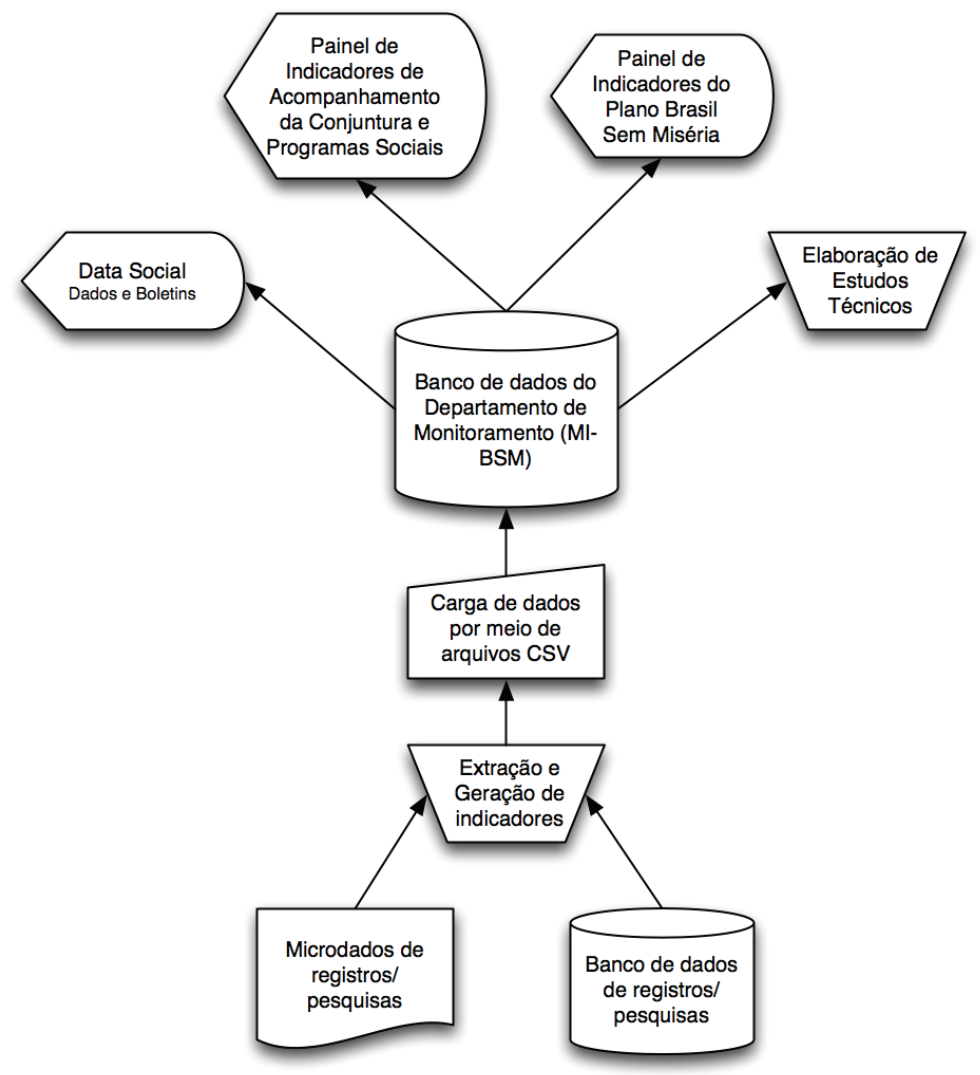

Atualmente o banco conta com mais de mento que estão disponibilizadas em três 1.600 variáveis oriundas de diversos replataformas: Data Social (Dados e Bolegistros administrativos de programas e de pesquisas do Sistema Estatístico Nacional.

\section{Aplicações de} tins); Painel de Indicadores de Acompanhamento da Conjuntura e de Programas Sociais; e Painel de Monitoramento do Plano Brasil Sem Miséria.

\section{Monitoramento na}

\section{SAGI}

O banco de dados do Departamento, por fim, alimenta as aplicações de monitora-

Além desse conjunto sistêmico de aplicações, o banco de dados também é ferramenta de consulta do Departamento para elaboração de estudos técnicos pontuais.

Revista Brasileira de Monitoramento e Avaliação | Número 5 | Janeiro-Junho de 2013

Monitoramento Analítico do Plano Brasil Sem Miséria e de Programas do Ministério do Desenvolvimento Social e Combate à Fome 


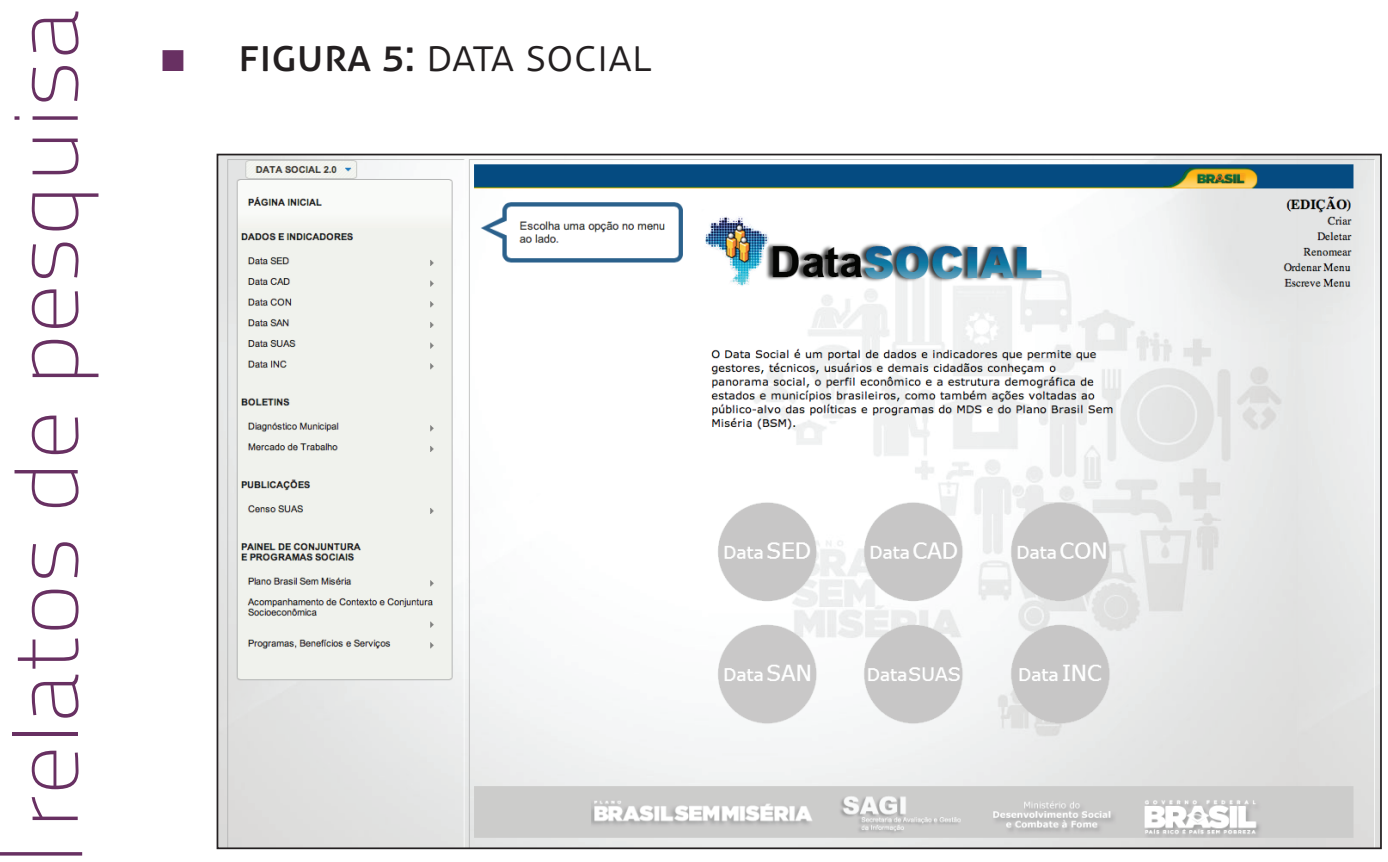

Apesar de o banco não ser exaustivo nas

para monitoramento das políticas e propossiblidades de recortes e análises que os microdados dos registros/pesquisas oferecem, o seu conjunto estruturado de indicadores otimiza o trabalho rotineiro de análise ao evitar redundância de processamento de indicadores recorrentes nos conteúdos trabalhados no âmbito do Ministério e do Plano Brasil Sem Miséria.

Uma das plataformas compostas pelos indicadores é o portal Data Social, que disponibiliza dados e indicadores para elaboração de diagnósticos atualizados e gramas do Ministério, além de informações de contexto social, demográfico e econômico de municípios, estados, regiões e Brasil. Dados e indicadores acerca da estrutura de gestão de programas, do dimensionamento e de características dos públicos-alvo das políticas, de insumos, entregas e resultados dos programas, serviços e ações do Ministério podem ser consultados nos componentes temáticos do portal, disponíveis para acesso de técnicos, gestores e população em geral na página da SAGI. 
FIGURA 6: BOLETINS MUNICIPAIS

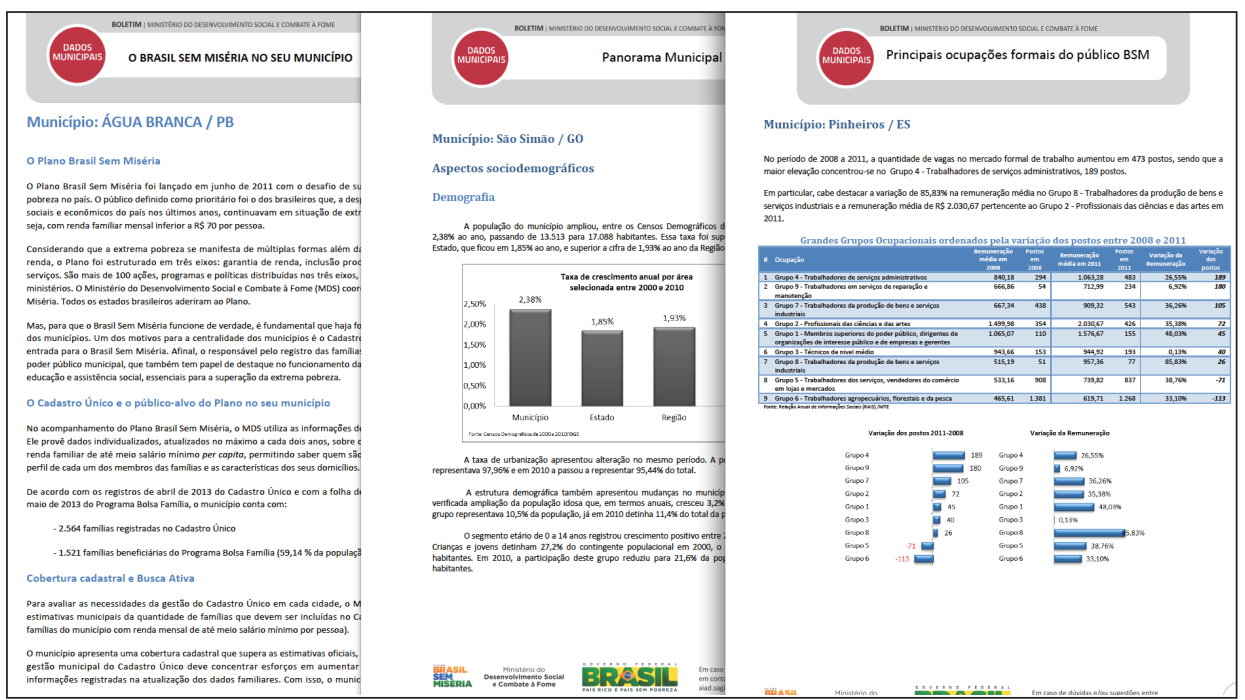

- OUADRO 2: RELAÇÃO DE BOLETINS PRODUZIDOS PELO DEPARTAMENTO ATÉ JULHO DE 2013

\section{BOLETIM}

Brasil Sem Miséria no seu Município

Subsídios para elaboração do PPA Municipal

Panorama Municipal segundo Censo Demográfico 2010

Extrema Pobreza segundo Censo Demográfico 2010

Diagnóstico Socioterritorial

Elaborando um Diagnóstico para Gestão Sugestões para o gestor elaborar um Diagnóstico para Gestão de Políticas e PrograMunicipal

Mercado de Trabalho segundo o Censo Demográfico 2010

Dinâmica das Ocupações Formais segundo RAIS

Inclusão Produtiva segundo Censo SUAS

Vulnerabilidade Social e Juventude Negra

Dinâmica de admissões em ocupações formais segundo CAGED

\section{CONTEÚDO}

Conjunto de indicadores referentes às ações que compõem o Plano Brasil Sem Miséria

Conjunto básico de indicadores para subsidiar o gestor municipal na elaboração do Plano Plurianual 2014-2017

Conjunto básico de indicadores para subsidiar a elaboração de diagnósticos municipais

Análise da população em situação de extrema pobreza no município

Conjunto básico de indicadores acerca de características demográficas, econômicas e sociais dos municípios para elaboração de um diagnóstico situacional que sirva de aporte à atuação da Assistência Social

mas Sociais em Âmbito Municipal

Distribuição das pessoas ocupadas no mercado de trabalho

Análise da evolução de vagas no mercado formal de trabalho para gestão e planejamento de oferta de cursos de qualificação

Análise da oferta de cursos por parte da gestão de Assistência Social e ações de geração de trabalho e renda e qualificação profissional

Indicadores demográficos, mercado de trabalho e vulnerabilidade social da juventude negra para suporte à atuação municipal no Plano Juventude Viva e Plano Brasil Sem Miséria

Apresentação das ocupações que mais geraram empregos nos municípios no primeiro semestre de 2013 para fins de subsidiar a definição dos cursos a serem ofertados pelo PRONATEC FIC Brasil Sem Miséria

Revista Brasileira de Monitoramento e Avaliação | Número 5 | Janeiro-Junho de 2013

Monitoramento Analítico do Plano Brasil Sem Miséria e de Programas do Ministério do Desenvolvimento Social e Combate à Fome 
A plataforma está dividida em seis conjuntos temáticos de indicadores: Data SED (principais dados e indicadores da área social, econômica e demográfica), Data CAD (dados do Cadastro Único para Programas Sociais e do Programa Bolsa Família), Data CON (dados sobre as condicionalidades de educação e saúde de beneficiários do Programa Bolsa Família), Data SAN (dados sobre contexto e programas de Segurança Alimentar e Nutricional), Data SUAS (dados sobre equipamentos, recursos humanos e serviços da Assistência Social) e Data INC (dados sobre mercado de trabalho e ações em Inclusão Produtiva).
Além dos indicadores, a aplicação disponibiliza boletins municipais temáticos para os 5.570 municípios brasileiros com conteúdos analíticos textuais e gráficos. Os boletins foram desenvolvidos no Departamento por meio de programações que estabeleciam relações condicionais entre indicadores e conteúdos textuais, possibilitando a geração automatizada de relatórios municipais personalizados. Neste sentido, a estruturação documentada de indicadores/variáveis do banco de dados do Departamento foi crucial para fomentar a produção de boletins analíticos automatizados.

\section{- FIGURA 7: PAINEL DE INDICADORES DE ACOMPANHAMENTO DA CONJUNTURA E DE PROGRAMAS SOCIAIS (PIC)}

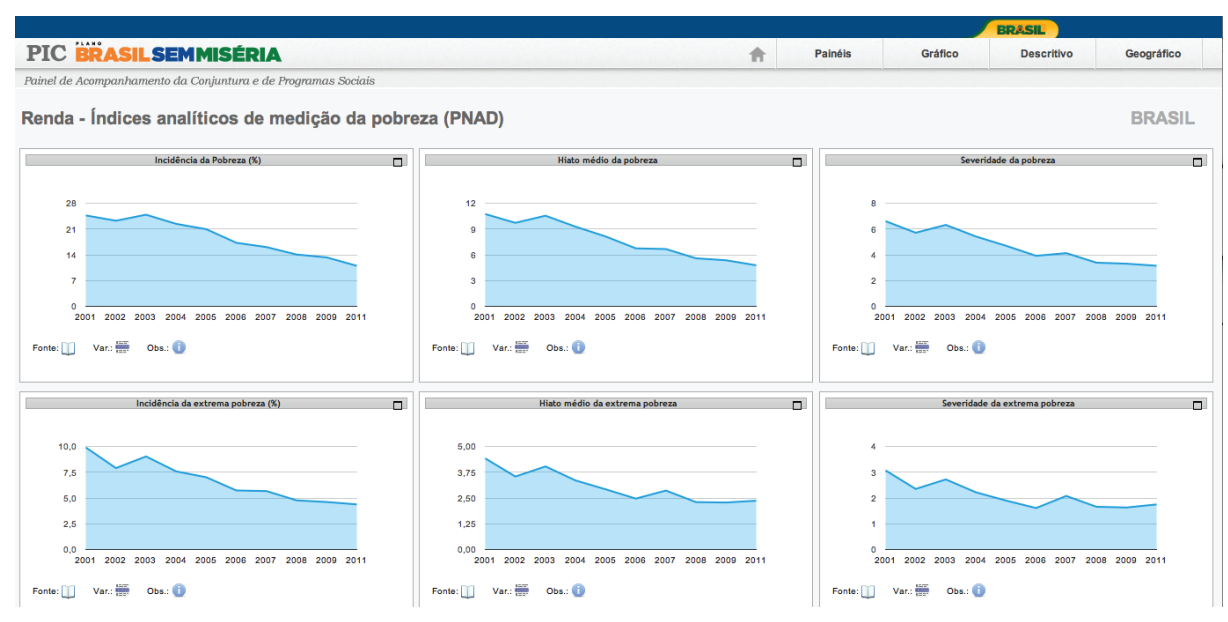


Integrado à plataforma do Data Social também se encontra o Painel de Indicadores de Acompanhamento da Conjuntura e de Programas Sociais, que também pode ser acessado diretamente do site institucional da SAGI. Esta aplicação apresenta painéis de indicadores de conjuntura relacionados à educação, ao mercado de trabalho, aos indicadores analíticos de desigualdade e pobreza e uma síntese de indicadores de programas, benefícios e serviços gestados pelo Ministério. Estes painéis têm o intuito de captar/dimensionar os esforços realizados pelo Ministério e os efeitos alcançados.
Por fim, o Departamento desenvolve e alimenta a aplicação Painel de Indicadores de Monitoramento do Plano Brasil Sem Miséria (MONIB) para o acompanhamento gerencial e analítico das ações do Plano em nível nacional, regional, estadual e municipal, dispondo de indicadores específicos de entregas e de seus efeitos. Conta com as mesmas funcionalidades do Painel de Indicadores de Acompanhamento da Conjuntura e de Programas Sociais, diferenciando-se na composição de indicadores e na inclusão de sistema de sinaleiras nas tabulações conforme intervalos de variação percentual dos indicadores no tempo.

\section{FIGURA 8: PAINEL DE INDICADORES DE MONITORAMENTO DO PLANO}

BRASIL SEM MISÉRIA (MONIB)

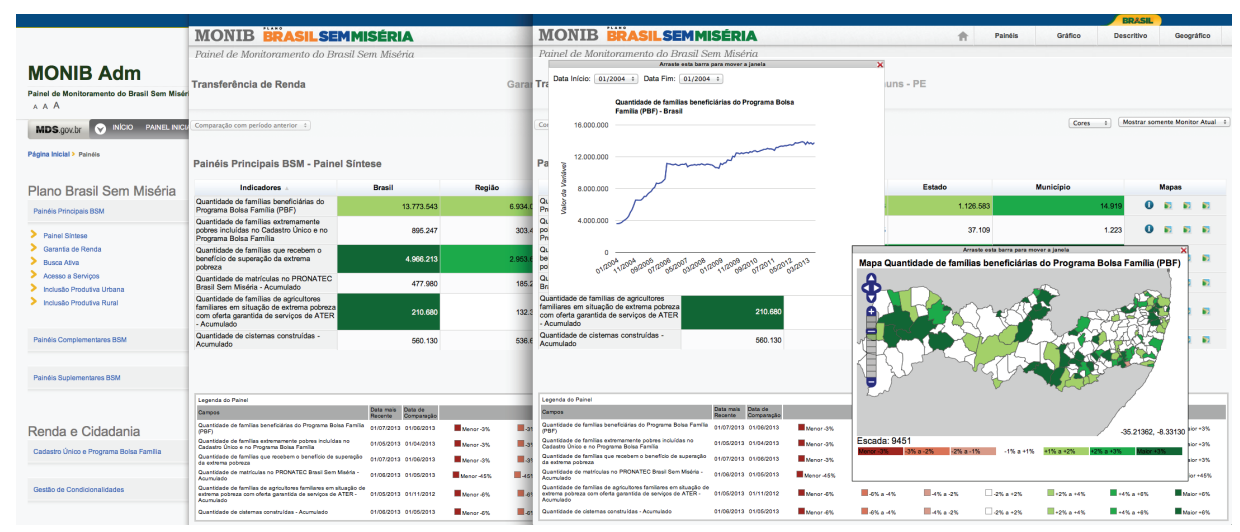


Um aspecto importante a ser elucidado sobre o $\mathrm{MONIB}^{2}$ é relativo à crescente introdução de indicadores construídos a partir da integração de dados provenientes do Cadastro Único com outras fontes de informação de registros de programas governamentais. De forma incremental, o Departamento vem construindo indicadores que tenham a especificidade que o desenho do Plano exige a partir de sua estruturação nos eixos de transferência de renda, acesso a serviços e inclusão produtiva. Indicadores que permitam a captação das interações de cobertura das ações destes eixos, especialmente as focalizadas exclusivamente nas famílias extremamente pobres, são de crucial importância para o monitoramento de uma atuação intersetorial sistêmica como a do Plano.

A integração de registros administrativos individualizados de diversos sistemas de gestão das ações e programas do Plano tem sido realizada a partir do Cadastro Único para Programas Sociais. Por meio de técnicas de construção de match codes, o Departamento integra e computa indicadores de acesso à formalização do emprego/empreendimento, assim como de acesso a programas e serviços sociais setoriais do público cadastrado e beneficiário do Programa Bolsa Família.

Este trabalho, para além do cômputo tempestivo de indicadores, tem possibilitado a realização de estudos longitudinais do público cadastrado, permitindo identificar fatores relacionados às políticas sociais que contribuem para o alcance de resultados esperados do Plano, como a melhoria de indicadores de educação, saúde, qualificação profissional e condições de inserção no mercado de trabalho. 


\section{Considerações finais}

O presente relato objetivou apresentar experiência diária de incorporação da sisuma descrição panorâmica da sistemática de produção e disseminação dos temática de monitoramento à gestão no âmbito do MDS. principais indicadores de monitoramento relativos aos programas e ações do Plano Brasil Sem Miséria e do Ministério de Desenvolvimento Social e Combate à Fome organizados pelo Departamento de Monitoramento. Trata-se de um esforço documental de um trabalho rotineiro e característico da tempestividade que a atividade de monitoramento exige. Todas as aplicações aqui descritas estão disponíveis no site institucional da $\mathrm{SAGI}^{3} \mathrm{e}$ seus aprimoramentos, do ponto de vista de funcionalidades e conteúdos, se incrementam conforme a consolidação da

Por fim, é importante destacar que só é possível a manutenção e consolidação de sistemáticas de monitoramento quando seus conteúdos e funcionalidades se adequam às necessidades dos gestores dos programas. Não há receita única para a construção de sistemas de monitoramento de políticas públicas, mas, sem dúvida, tão importante quanto trabalhar com indicadores consistentes, é a premissa constante de que a atividade deve objetivar tempestivamente o subsídio à tomada de decisão na gestão de ações e programas.

2 Recomenda-se a leitura do ETEC nº 01/2013 - MONIB: Painel de Indicadores de Monitoramento do Plano Brasil Sem Miséria - concepção e funcionalidades para maior conhecimento da aplicação.

Revista Brasileira de Monitoramento e Avaliação | Número 5 | Janeiro-Junho de 2013 